\title{
Reducing Hubness for Kernel Regression
}

\author{
Kazuo Hara ${ }^{1}$, Ikumi Suzuki ${ }^{2}$, Kei Kobayashi², Kenji Fukumizu ${ }^{2}$, and \\ Miloš Radovanović ${ }^{3}$ \\ 1 National Institute of Genetics, Mishima, Shizuoka, Japan \\ 2 The Institute of Statistical Mathematics, Tachikawa, Tokyo, Japan \\ 3 University of Novi Sad, Novi Sad, Serbia
}

\begin{abstract}
In this paper, we point out that hubness - some samples in a high-dimensional dataset emerge as hubs that are similar to many other samples - influences the performance of kernel regression. Because the dimension of feature spaces induced by kernels is usually very high, hubness occurs, giving rise to the problem of multicollinearity, which is known as a cause of instability of regression results. We propose hubness-reduced kernels for kernel regression as an extension of a previous approach for $k \mathrm{NN}$ classification that reduces spatial centrality to eliminate hubness.
\end{abstract}

\section{Introduction}

Recently, hubness, a phenomenon occurring in high-dimensional datasets as a result of curse of dimensionality [5], has attracted the attention of researchers in the artificial intelligence community, especially for data mining and machine learning. For instance, a new clustering algorithm was presented by taking advantage of hubness [9]. The performance of $k$-nearest-neighbor $(k \mathrm{NN})$ classification was improved by eliminating hubness $[7,6,8,3]$.

In this paper, we point out that the hubness influences the performance of kernel regression as well. Because the dimension of feature spaces induced by kernels is usually very high, hubness occurs. Therefore, in the learning phase of kernel regression, hubs in training samples that are similar to many other training samples provide highly correlated information to the learning model. The problem caused by such correlation of input variables is known as multicollinearity, which degrades the generalization error on test samples $[4,1]$.

We then propose hubness-reduced kernels for kernel regression as an extension of localized centering [3], a technique for $k \mathrm{NN}$ classification, which transforms similarity measures to reduce spatial centrality to get rid of hubness.

\section{Multicollinearity in Kernel Regression}

Let us assume that $n$ pairs of input object and a scalar output value $\left\{x_{i}, y_{i}\right\}_{i=1}^{n}$ are given as training samples, and that the goal is to predict the output $y_{\text {test }}$ for a new input $x_{\text {test }}$. In ordinary linear regression, an input object $x$ is represented as a $d$-dimensional vector. More formally,

$$
x \mapsto f(x)=\left(f_{1}(x), \ldots, f_{d}(x)\right)^{\mathrm{T}},
$$


where a set of $d$ functions $\left\{f_{i}(x)\right\}_{i=1}^{d}$ are ad-hoc feature extractors designed by domain experts. Then, weight $w_{f}$ of the functions, which is a $d$-dimensional vector, is determined using training samples to minimize the least-squares loss calculated as $\sum_{i=1}^{n}\left(y_{i}-w_{f}^{\mathrm{T}} f\left(x_{i}\right)\right)^{2}$. Also, the output $y_{\text {test }}$ for a new input $x_{\text {test }}$ is predicted as $\hat{y}_{\text {test }}=w_{f}^{\mathrm{T}} f\left(x_{\text {test }}\right)$.

In the situation described above, multicollinearity is related to cases in which the functions $\left\{f_{i}(x)\right\}_{i=1}^{d}$ are highly correlated, or to cases in which a function extracts feature values from input objects in a very similar way to that of the other functions. In such a case, a problem occurs by which the prediction becomes less reliable because the weight $w_{f}$ tends to be overfitted to noisy training samples $[4,1]$.

In contrast, with kernel regression, an input object $x$ is mapped to an $n$ dimensional vector, such that

$$
x \mapsto k(x)=\left(k_{1}(x), \ldots, k_{n}(x)\right)^{\mathrm{T}},
$$

where $\left\{k_{i}(x)\right\}_{i=1}^{n}$ are kernel functions that give similarity between input $x$ and a training object $x_{i}$. Some examples include $k_{i}(x)=\left\langle f(x), f\left(x_{i}\right)\right\rangle$ for linear kernels, and $k_{i}(x)=\exp \left(-\frac{1}{2 \gamma^{2}}\left\|f(x)-f\left(x_{i}\right)\right\|^{2}\right)$ for Gaussian kernels. These kernel functions $k_{i}(x)$ take larger values for input objects lying in the neighbor of the training object $x_{i}$, and smaller values for other input objects. If neighbor objects are different for each of the training objects $\left\{x_{i}\right\}_{i=1}^{n}$, then corresponding kernel functions $\left\{k_{i}(x)\right\}_{i=1}^{n}$ take large values for different objects. Therefore, no correlation emerges between the kernel functions.

However, because a vector space (i.e., a reproducing kernel Hilbert space) induced by kernels is usually high-dimensional, hub objects that are similar to many other objects tend to occur, and the kernel function $k_{h}(x)$ corresponding to a hub object $x_{h}$ gives large values for many objects. This fact implies that $k_{h}(x)$ correlates with other kernel functions, and thereby produces multicollinearity.

\section{Hubness in Kernel-Induced Spaces}

The phenomenon of hubness is known to emerge when the nearest neighbors (NNs) in a high-dimensional dataset are considered [5]. Let $D \subset \mathbb{R}^{d}$ be a dataset in $d$-dimensional space and let $N_{k}(x)$ denote the number of times a sample $x \in D$ occurs in the $k \mathrm{NNs}$ of other samples in $D$, under some similarity measure. When the dimension is high, the shape of the $N_{k}$ distribution skews to the right. A small number of samples take large $N_{k}$ values. Such samples, similar to many other samples, are called hubs. This phenomenon is called hubness.

We next demonstrate the emergence of hubness using artificial data. we generate a dataset from a mixture of two Gaussian distributions with sample size $n=1000$ and dimension $d=1000$. Specifically, we generate 500 samples each from i.i.d. Gaussian $(\mathbf{0}, \mathbf{I})$ and i.i.d. Gaussian $(\mathbf{1}, \mathbf{I})$, where $\mathbf{0}, \mathbf{1}$ respectively denote $d$-dimensional vectors of zeros and ones and $\mathbf{I}$ is the $d \times d$ identity matrix.

To determine $k \mathrm{NN}$ samples, we use a positive definite kernel that is equal to the inner-product on a Hilbert space induced by the kernel. Note that each 


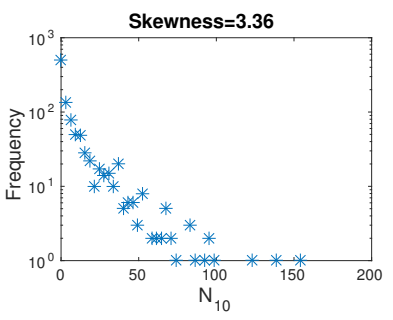

(a) Hubness exists.

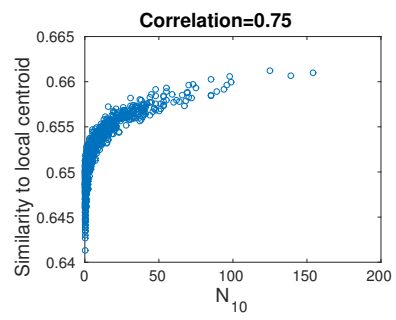

(b) Spatial centrality.

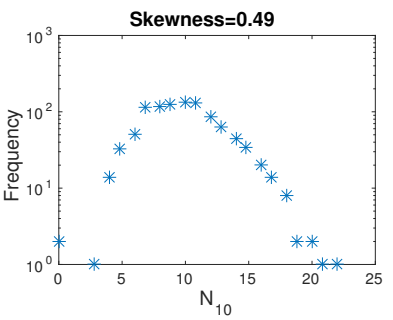

(c) Hubness disappears.

Fig. 1. (a) Hubness occurs: samples with a large $N_{10}$ value occur, and the $N_{10}$ distribution is skewed to the right. (b) Correlation between the $N_{10}$ value and the similarity to the local centroid is strong. (c) Hubness is reduced (lower $N_{10}$ value and smaller skewness) by the transformation according to Equation (7).

sample $x \in D$ is mapped to the Hilbert space according to a function denoted as $\phi(x)$. Here, we use a Gaussian kernel, ${ }^{1}$ where similarity between two samples $x_{i}, x_{j} \in D$ is given as

$$
K\left(x_{i}, x_{j}\right)=\left\langle\phi\left(x_{i}\right), \phi\left(x_{j}\right)\right\rangle=\exp \left(-\frac{1}{2 \gamma^{2}}\left\|x_{i}-x_{j}\right\|^{2}\right)
$$

with a deviation parameter $\gamma$ set as the median of pairwise distances among samples in $D$. The distribution of $N_{10}$ is shown in Figure 1(a). We can observe the presence of hubs, i.e., samples with a particularly large $N_{10}$ values.

Following Radovanović et al. [5], we evaluate the degree of hubness by the skewness of the $N_{k}$ distribution. A large skewness indicates strong hubness in a dataset. Indeed, skewness is large (i.e., 3.36) in Figure 1(a).

\subsection{Origin of Hubness: Spatial Centrality}

For the artificial dataset described above, we form a scatter plot of samples with respect to the $N_{10}$ value and the similarity to the local centroid (Figure 1(b)). A local centroid is defined for each sample $\phi(x)$ as

$$
c_{\kappa}(\phi(x)) \equiv \frac{1}{\kappa} \sum_{\phi\left(x^{\prime}\right) \in \kappa \mathrm{NN}(\phi(x))} \phi\left(x^{\prime}\right),
$$

which is the mean of the $\kappa$-nearest neighbor samples of $\phi(x)$ under some local neighborhood size $\kappa \in[1, n-1]$ [3]. It is noteworthy that the local centroid is not always obtained explicitly using Equation (4), because it is computed not in the original $d$-dimensional space but in a space induced by the kernel that defines $K(\cdot, \cdot)$. Therefore, the similarity to the local centroid is calculated as

$$
\left\langle\phi(x), c_{\kappa}(\phi(x))\right\rangle=\frac{1}{\kappa} \sum_{\phi\left(x^{\prime}\right) \in \kappa \mathrm{NN}(\phi(x))} K\left(x, x^{\prime}\right) .
$$

\footnotetext{
${ }^{1}$ Gaussian kernel is a shift-invariant kernel, where the NN based on the inner-product $\left\langle\phi\left(x_{i}\right), \phi\left(x_{j}\right)\right\rangle$ is equivalent to that based on the norm $\left\|x_{i}-x_{j}\right\|$.
} 
In the dataset generated from two Gaussians, strong correlation exists between the $N_{10}$ value and the similarity to the local centroid $(\kappa=20)$ as shown in Figure 1(b). This is called spatial centrality of the dataset.

\section{Reducing Hubness for Kernel Regression}

Because the existence of spatial centrality is considered to be an ingredient of hubness [5], hubness is expected to be suppressed by removing the spatial centrality. Following this idea, Hara et al. [3] proposed a hubness-reduction method called localized centering for $k \mathrm{NN}$ classification. The method transforms the similarity measure such that the transformed similarity does not generate spatial centrality. The transformation is given by subtracting similarity to the local centroid in Equation (5) from the original similarity in Equation (3), such that

$$
\begin{aligned}
\operatorname{Sim}^{\mathrm{LCENT}}\left(x_{i}, x_{j}\right) & \equiv\left\langle\phi\left(x_{i}\right), \phi\left(x_{j}\right)\right\rangle-\left\langle\phi\left(x_{j}\right), c_{\kappa}\left(\phi\left(x_{j}\right)\right\rangle\right. \\
& =K\left(x_{i}, x_{j}\right)-\frac{1}{\kappa} \sum_{\phi\left(x^{\prime}\right) \in \kappa \mathrm{NN}\left(\phi\left(x_{j}\right)\right)} K\left(x_{j}, x^{\prime}\right) .
\end{aligned}
$$

After this transformation, the similarity to the local centroid for any database sample $x_{j}$ becomes the same because substituting $\phi\left(x_{i}\right)=c_{\kappa}\left(\phi\left(x_{j}\right)\right)$ in Equation (6) yields a constant value (i.e., zero). This fact indicates that no spatial centrality with respect to the local centroid exists after the transformation. Therefore, the hubness is expected to be reduced. Indeed, by applying the transformation to the dataset used to draw Figure 1, the skewness decreases from 3.36 (Figure 1(a)) to 0.49 (Figure 1(c)).

It should be noted that the resulting similarity measure $\operatorname{Sim}^{\mathrm{LCENT}}(\cdot, \cdot)$ is not symmetric with respect to $x_{i}$ and $x_{j}$. This does not matter for $k \mathrm{NN}$ classification because it is assumed that similarity is computed between two samples that have different roles, i.e., a query and a database sample. Indeed, $x_{i}$ and $x_{j}$ in Equation (6) respectively correspond to a query and a database sample.

We now propose hubness-reduced kernels by a symmetrization of Sim $^{\text {LCENT }}$, as follows.

$$
\begin{aligned}
& K^{\mathrm{HR}}\left(x_{i}, x_{j}\right) \equiv\left\langle\phi\left(x_{i}\right)-c_{\kappa}\left(\phi\left(x_{j}\right)\right), \phi\left(x_{j}\right)-c_{\kappa}\left(\phi\left(x_{i}\right)\right)\right\rangle \\
& =\left\langle\phi\left(x_{i}\right), \phi\left(x_{j}\right)\right\rangle-\left\langle\phi\left(x_{i}\right), c_{\kappa}\left(\phi\left(x_{i}\right)\right)\right\rangle-\left\langle\phi\left(x_{j}\right), c_{\kappa}\left(\phi\left(x_{j}\right)\right)\right\rangle+\left\langle c_{\kappa}\left(\phi\left(x_{i}\right)\right), c_{\kappa}\left(\phi\left(x_{j}\right)\right)\right\rangle \\
& =K\left(x_{i}, x_{j}\right)-\frac{1}{\kappa} \sum_{\phi\left(x^{\prime}\right)} K\left(x_{i}, x^{\prime}\right)-\frac{1}{\kappa} \sum_{\phi\left(x^{\prime \prime}\right)} K\left(x_{j}, x^{\prime \prime}\right)+\frac{1}{\kappa^{2}} \sum_{\phi\left(x^{\prime}\right) \phi\left(x^{\prime \prime}\right)} K\left(x^{\prime}, x^{\prime \prime}\right)
\end{aligned}
$$

The transformed kernels are not always positive definite. For such a case, we replace all negative eigenvalues of the transformed kernels with zeros. ${ }^{2}$

\footnotetext{
${ }^{2}$ It is important to pursue if this is a good way of fixing the non-positive definiteness as a future work.
} 


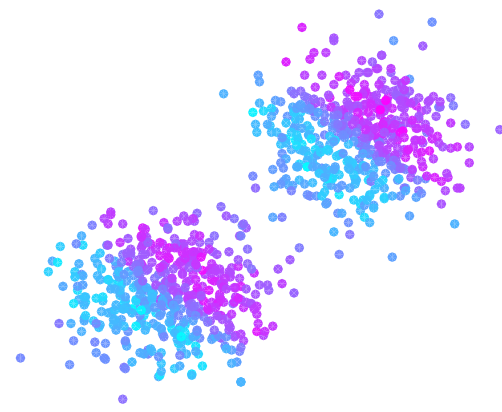

(a)

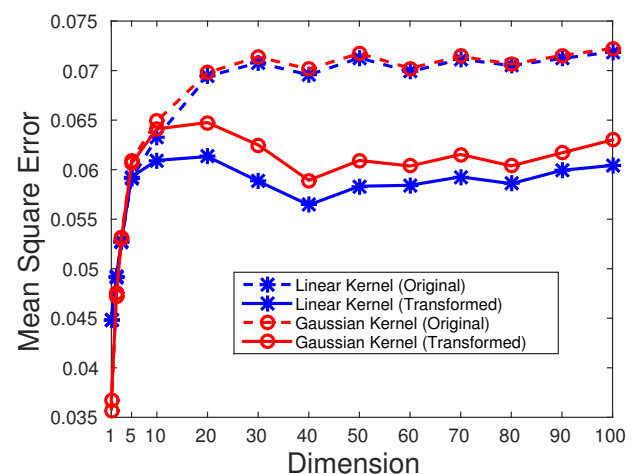

(b)

Fig. 2. (a) An illustration of the multi-centered dataset (generated with sample size $n=1000$, dimension $d=2$, and $\delta=4$ ). Red and blue points respectively correspond to samples with positive and negative outputs. (b) Mean squared error of kernel regression, using multi-centered datasets with $n=100, d \in[1,100]$, and $\delta=1$.

\section{Experiment}

We demonstrate that the proposed kernels $K^{\mathrm{HR}}$ reduce the correlation between input variables, i.e., multicollinearity. Thereby, they improve prediction accuracy. We used artificial data generated from a mixture of two Gaussian distributions ${ }^{3}$ with a fixed sample size $n=100$ and variation in dimension $d$ from 1 to 100 . More precisely, the dataset consisted of $\left\{x_{i}\right\}_{i=1}^{\frac{n}{2}}$ generated from i.i.d. $\operatorname{Gaussian}(\mathbf{0}, \mathbf{I})$ with output $y_{i}=\sin \left(z_{i}\right) \exp \left(-\left|z_{i}\right|\right)+\epsilon$, where $z_{i}=\left\langle x_{i}, \frac{1}{\|\mathbf{1}\|}\right\rangle$, and $\left\{x_{i}\right\}_{i=\frac{n}{2}+1}^{n}$ generated from i.i.d. $\operatorname{Gaussian}(\delta \mathbf{1}, \mathbf{I})$ with output $y_{i}=\sin \left(z_{i}\right) \exp \left(-\left|z_{i}\right|\right)+\epsilon$, where $\delta=1, z_{i}=\left\langle x_{i}-\delta \mathbf{1}, \frac{\mathbf{1}}{\|\mathbf{1}\|}\right\rangle$, and $\epsilon$ is noise generated from $\operatorname{Gaussian}\left(\mathbf{0}, \sigma^{2} \mathbf{I}\right)$ where $\sigma^{2}=0.01$. The dataset is illustrated in Figure 2(a).

In the manner described above, we generated samples of size $n$ each for training, validation, and testing. However, they were centered or shifted such that the mean of the training samples was zero.

For kernel regression, we used a linear kernel $K\left(x_{i}, x_{j}\right)=\left\langle x_{i}, x_{j}\right\rangle$ and a Gaussian kernel $K\left(x_{i}, x_{j}\right)=\exp \left(-\frac{1}{2 \gamma^{2}}\left\|x_{i}-x_{j}\right\|^{2}\right)$ with a deviation parameter $\gamma$ set as the median of pairwise distances among training samples, following Gretton et al. [2]. For each kernel, we tested the proposed transformation according to Equation (8).

To avoid overfitting to training samples, we used kernel ridge regression. Given a kernel matrix $K$ and a vector $y$ of output values with respect to training samples, the model parameter $\alpha$ was learned as a solution $\alpha=(K+\lambda I)^{-1} y$,

\footnotetext{
${ }^{3}$ We used multi-centered datasets, because the correlation between training samples is not reduced by centering, a common pre-processing to avoid multicollinearity.
} 
where $\lambda$ is a hyper-parameter of ridge regression. We selected $\lambda$ as well as the hyper-parameter $\kappa$ of the proposed method using validation samples.

The output $y_{t}$ of a test sample $x_{t}$ was predicted as $\hat{y}_{t}=\sum_{i=1}^{n} \alpha_{i} k\left(x_{i}, x_{t}\right)$ using training samples $\left\{x_{i}\right\}_{i=1}^{n}$. The methods were evaluated according to the mean squared error (MSE) $\left(y_{t}-\hat{y_{t}}\right)^{2}$ over the test samples.

For each setting of dimension $d$ ranging from 1 to 100 , we repeated the process described above 100 times. The average of the MSE obtained is shown in Figure 2(b).

Figure 2(b) shows that when the number of dimensions is large (i.e., more than 10), the proposed kernel transformation improves MSE for both linear and Gaussian kernels. The result suggests that hubness emerges in high-dimensional data, and affects kernel regression through the resulting multicollinearity. However, hubness, and hence the MSE can be reduced using our proposed kernels.

\section{Conclusion}

After pointing out that hubness gives rise to the multicollinearity problem, and that it therefore influences the performance of kernel regression, we proposed hubness-reduced kernels for kernel regression as an extension of a previous approach for $k \mathrm{NN}$ classification. We demonstrated that reduction of hubness produces an effect on kernel regression for multi-centered datasets.

\section{References}

1. Chatterjee, S., Hadi, A.S., Price, B.: Regression Analysis By Example. Wiley Series In Probability And Statistics, Wiley, New York (2000)

2. Gretton, A., Fukumizu, K., Teo, C., Song, L., Schölkopf, B., Smola, A.: A kernel statistical test of independence. In: Advances in neural information processing systems 20. pp. 585-592 (2008)

3. Hara, K., Suzuki, I., Shimbo, M., Kobayashi, K., Fukumizu, K., Radovanović, M.: Localized centering: Reducing hubness in large-sample data. In: AAAI (2015)

4. Montgomery, D.C., Peck, E.: Introduction to linear regression analysis. WileyInterscience Publication, John Wiley \& sons, New York (1992)

5. Radovanović, M., Nanopoulos, A., Ivanović, M.: Hubs in space: Popular nearest neighbors in high-dimensional data. Journal of Machine Learning Research 11, 2487$2531(2010)$

6. Schnitzer, D., Flexer, A., Schedl, M., Widmer, G.: Local and global scaling reduce hubs in space. Journal of Machine Learning Research 13(1), 2871-2902 (2012)

7. Suzuki, I., Hara, K., Shimbo, M., Matsumoto, Y., Saerens, M.: Investigating the effectiveness of laplacian-based kernels in hub reduction. In: AAAI (2012)

8. Suzuki, I., Hara, K., Shimbo, M., Saerens, M., Fukumizu, K.: Centering similarity measures to reduce hubs. In: EMNLP. pp. 613-623 (2013)

9. Tomasev, N., Radovanovic, M., Mladenic, D., Ivanovic, M.: The role of hubness in clustering high-dimensional data. IEEE Transactions on Knowledge and Data Engineering 26(3), 739-751 (2014) 